\title{
The Pumapaint Project: Long Term Usage Trends And The Move To Three Dimensions
}

\author{
Matthew R. Stein \\ Associate Professor of Engineering \\ Roger Williams University \\ Bristol, RI 02809 \\ mstein@rwu.edu
}

\author{
Christopher P. Madden \\ Graduate Student \\ University of Connecticut \\ Christopher.madden@uconn.edu
}

\begin{abstract}
Online since the summer of 1998, the PumaPaint project can be considered an elder statesman of networked robotics. Although never a profound technical achievement to attach a PUMA robot to a network, the extended period of operation permits interesting observations of long-term usage trends, comparison of operation through several generations of user interface and a voluminous body of artwork to consider. This paper will present a discussion of long-term usage trends of the PumaPaint project over six years of operation at two locations. We will present a study of the most interesting artwork produced in the last two years and discuss trends from the perspective of the canvas. We will also present a case study of a recent phenomenon: the appearance of a dedicated and persistent vandal who returned to the site nightly for a period of several months. The second section of the paper will detail our current project: moving the PumaPaint concept to three dimensions. We are continuing the theme of artistic creation, this time allowing web users to model clay. We have partially completed construction on a pair of opposing anthropomorphic hands with force sensing capability and expect to have these operational by spring 2005. This paper will present the "RoboSculpt" concept, detail the design and proposed user interface and present the progress to date.
\end{abstract}

Keywords-Networked robotics, telerobotics, kinesthetic feedback

\section{INTRODUCTION}

The PumaPaint Project $[1,2,3,4,5]$ is an online robot that allows World Wide Web users to remotely create original artwork. The original site at Wilkes University [6] operated from June of 1998 to March of 2000 with approximately 25,000 unique-addressed machines downloading the interface to produce about 500 canvases. The newer site at Roger Williams University has been operational since August 2002 with about 10,000 users to over the two-year period ending in August 2004. Despite its relative longevity, the PumaPaint site has many limitations. Users can create paintings by commanding the PumaPaint robot, but this manipulation is largely symbolic. The highest level of direct manipulation a user can exert on the robot is commanding the location of brush strokes and the pressure the brush exerts on the canvas.

Among the most interesting aspects of PumaPaint is the relationship to artistic creation. The paintings users create are not only an experiment in telepresence, but also an experiment in painting. Users are invited to an act of spontaneous creation, and many users accept this opportunity and choose artistic expression as their form of communication.
We are continuing the theme of artistic creation with a new networked robot experience. We are seeking to present users to opportunity to model clay with a pair of mutually opposing anthropomorphic hands. Again, the technical challenges associated with creating the experience will be mostly invisible to the user. Akin to the blank canvas presented to the PumaPaint user, the new site, tentatively named "RoboSculpt", will present the user a unshaped lump of clay and invite him or her to sculpt.

\section{PUMAPAINT SITE EXPERIENCES}

The PumaPaint site has been online for about four of the last six years. We revived it in August 2002 at Roger Williams University after a two years recreating the infrastructure, and it has operated continuously until the time of this writing (September 2004). It should be understood that we are using "continuously" in a loose sense, and "as much as we found possible" would perhaps be most accurate. The site was offline for long weekends and holidays throughout the period, as paintbrushes and paper need changing daily. Over three or more days of unsupervised operation the paper weakens and is torn or the brushes are dropped.

Figure 1 show website access statistics for the first year of operation of the Wilkes University site and the entire period of operation of the Roger Williams University (RWU) site. Note there is a roughly three-year gap from July 1999 to August 2002 when the site was being recreated at RWU. Although interest waxes and wanes, Figure 1 shows activity of the RWU to be comparable to the Wilkes site during the first year when the site was novel. Spikes in accesses usually can be traced to media coverage events or listing on prominent web pages. For example, the January '03 spike can be traced to a brief mention on WebTV.

Figure 2 shows interface accesses by month, limited to the RWU site. The three bars are respectively "Interface", "PumaPaint class", and "Javax swing error". Access to the interface occurs when a user follows the "Click here to try" link. A comparison to Figure 1 shows there are roughly half the number of accesses of the main page, indicating that only half of visitors to the main page care to "click here to try". The second two bars are respectively successful downloads of the java interface and failures due to incompatibility with Java2/swing. Roughly speaking the latter two should add up to the former, as most attempts to load the interface will either succeed or fail due to browser incompatibility with Swing. 
Although slightly difficult to see in the space provided, there seems to be a trend towards fewer potential users encountering the browser incompatibility problem. This may be attributable to Java2/swing compatibility in newer versions of Internet Explorer, but one can also see there are still many users encountering this problem.

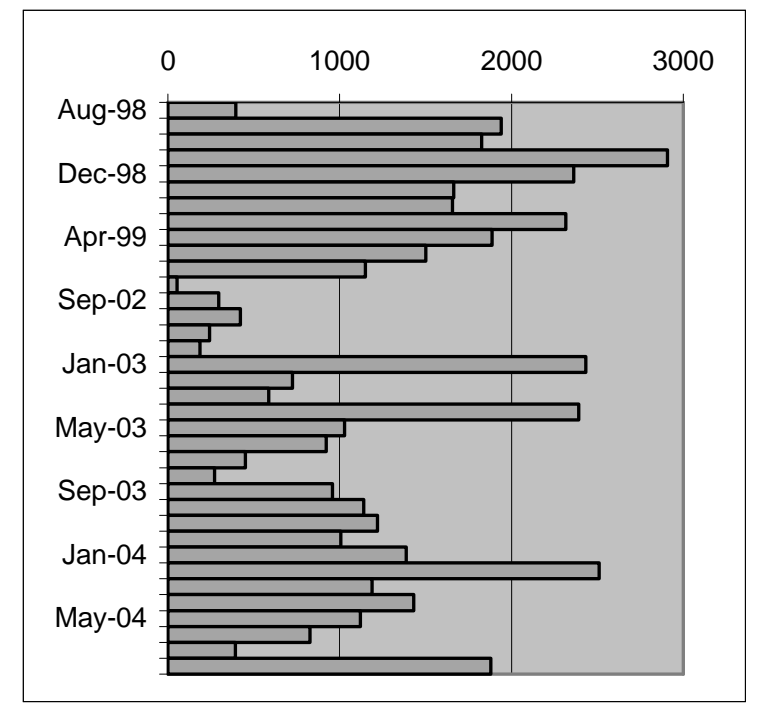

Figure 1. Accesses of the main PumaPaint site by month.

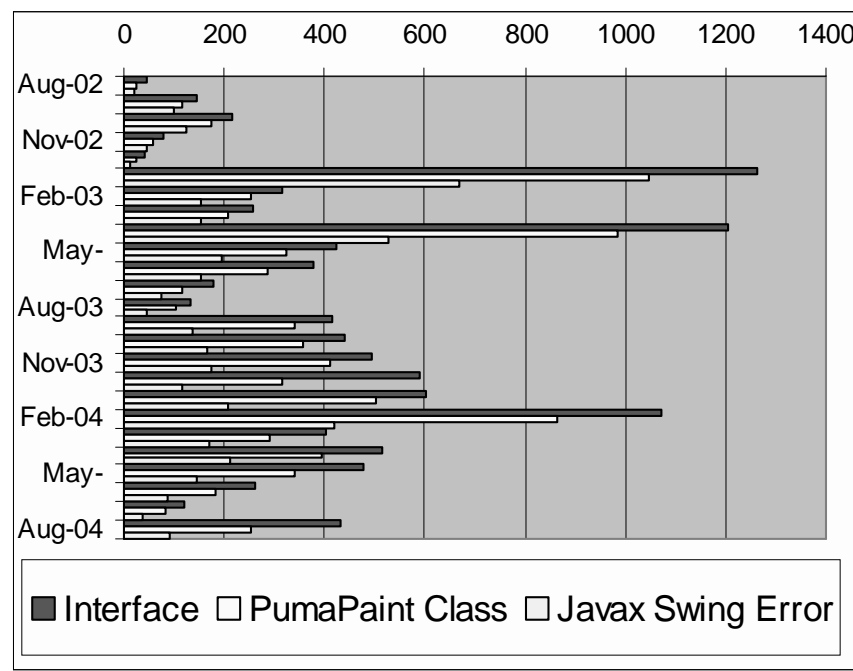

Figure 2. Accesses of the Java interface by month.

\begin{tabular}{|c|r|}
\hline Listing Site or Search Engine & References \\
\hline Google & 5160 \\
Yahoo & 1554 \\
Nasa Telerobotics & 1397 \\
Robots.net & 952 \\
\hline Excite & 174 \\
MSN search & 172 \\
AOL search & 108 \\
\hline
\end{tabular}

Figure 3. Referring sites.

\begin{tabular}{|c|r|}
\hline Main Page & 44672 \\
\hline Interface & 20018 \\
\hline PumaPaint Class & 15615 \\
\hline Javax Swing Error & 4243 \\
\hline
\end{tabular}

Figure 4. Totals for entire period of operation.

Figures 3 and 4 show numeric totals for accesses of each of the categories discussed. It can be seen that about 20,000 users have attempted to download the Java interface and about 15,000 succeeded. We believe this number speaks volumes for the robustness and longevity of the PumaPaint site. Although our referrer log was not configured with date information, we are able to make raw counts of referring sites. We see that Google searches are by far the most means of finding the site.

\section{THE DEDIACTED VANDAL}

A new phenomenon for the PumaPaint site occurred in late 2003 to early 2004 with the appearance of a dedicated and persistent vandal. This individual would visit the site every night, and became very adept and wreaking physical havoc on the robot. We were fascinated by this user, and spent hours watching him or her use the site.

The user connected from the same AOL server on every occasion, and so we referred to this user as the "AOL User" on the web page and in the image gallery. It would have been relatively easy to prevent the connection to the robot. However, one primary motivation for establishing the PumaPaint site was to see what the world would do with it. These attacks were something the world was doing with it, so we were more interested in seeing what would happen than defeating the intentions of the user. Our own curiosity and adherence to this principle did have some negative consequences. The site was down two months for maintenance immediately following the period of greatest activity of this user. As some of the mechanical problems were related to overheating of the motors, it may be fair to suggest the AOL User wore the robot out with nightly visits.

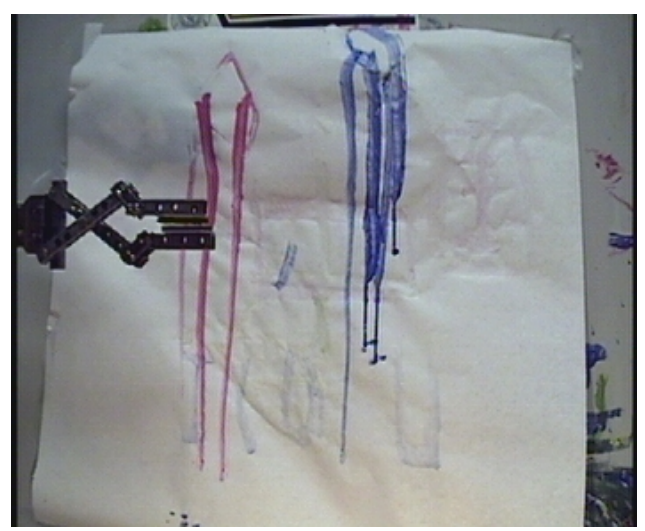

Figure 5. First appearance of the AOL user.

The first apparent appearance of the AOL user is the canvas shown in Figure 5 from November $17^{\text {th }}, 2003$. There is little atypical about the canvas but one can note blotches of paint running down the front. These blotches are indicative of a 
successful attempt to tear down the paper. However, the paper falling down is a common occurrence, so we thought nothing of it at the time. The next apparent canvas from the AOL user is shown in Figure 6. Here we can see the pattern of long vertical strokes that would become the signature of the AOL user. One can speculate that the user experimented with repetitive strokes in the art of painting, and either discovered or devised a means of producing them in great number.

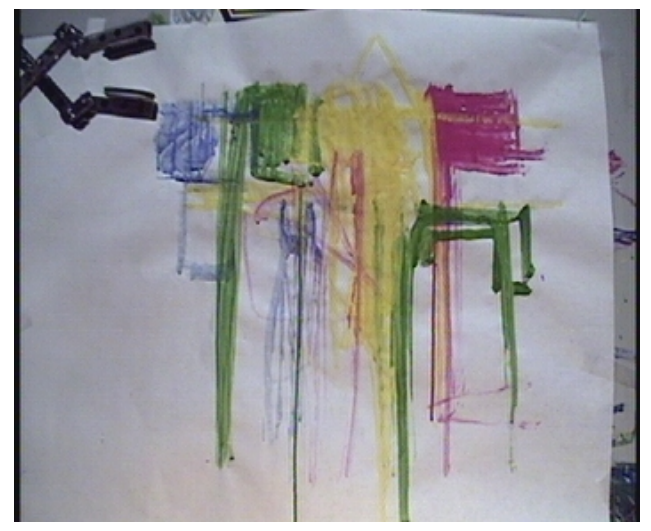

Figure 6. Initial indications of vertical strokes.

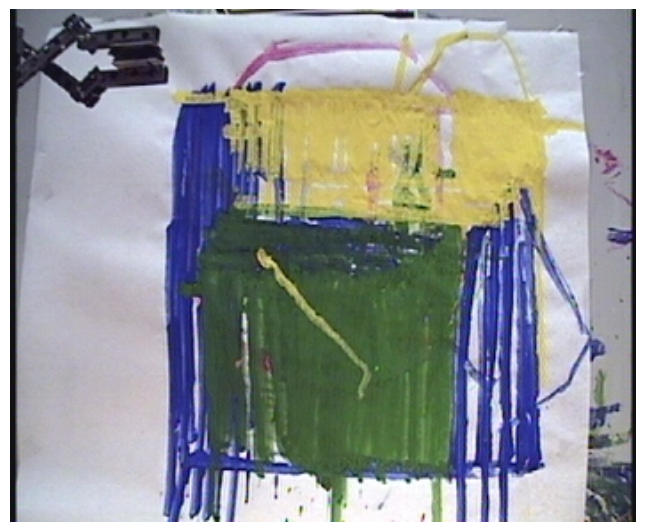

Figure 7. First occurance of voluminous and regular vertical strokes.

We first perceived that these canvases were being intentional produced by the same user on December $20^{\text {th }}$, with the canvas shown in Figure 7 . The canvas shows an unusual volume of paint. This much paint can only be produced by hundreds of strokes and a user spending more than an hour creating them. Our fist attempt was to encourage the user, reassuring him or here that we were intrigued rather than angered by his or her use of the site. Beneath the regular appearance in the image gallery we placed the following text:

"Very interesting. This may be the first known evidence of a dedicated prankster. This canvas appears to be the same AOL user as Nov 18, and the canvas is covered by vertical strokes. The robot paints these with metronome regularity, suggesting an automated loop or script rather than simply repetitively stroking on the interface. It would be fairly straightforward to connect up to the PumaPaint socket and issue commands, or perhaps this is some Windows macro using the interface. I would love to know if this is an ingenious break-in, can you please clue me in, even anonymously? I'm just one guy at a small university, so I have no way of tracking you down or any authority to punish. After all, this is a public domain site and you are using it in a public domain way. We await your next move."

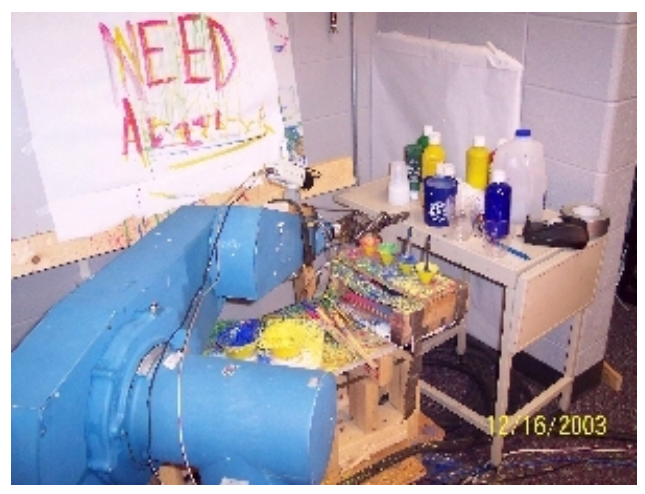

Figure 8. Serious "crash". Robot driven to singularity and easel knocked over.

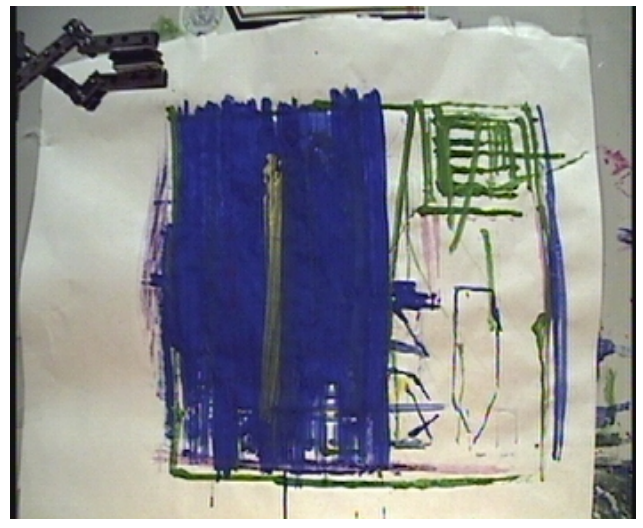

Figure 9. Return one month after first appearance.

The next evening the robot was "crashed" with a joint2-3 singularity, as shown in Figure 8, and the easel was knocked over. This was the first of its kind occurance for the PumaPaint site and can only be caused by moving the end effector very rapidly. We began to suspect the user had hacked into the robot controller and was swinging the arm around wildly. If this were truly the case we would probably have to shut the site down for safety reasons. Again, we placed the following message in the image gallery including the following text:

“... This has been very interesting, if you are the prankster; we are tightening our motion limits and awaiting your next move with great anticipation. Again, we find this most interesting and would love to be certain this is intentional and not just our interface breaking."

The next evening the AOL User returned and produced of the same robot behavior, as shown in Figure 9. We observed the robot carefully during the more than one hour session in control of the robot. The user caused the robot to make very repetitive vertical and horizontal strokes as can be seen from the bottom and right edges of the canvas. Also, a "dip" 
command is issued to the robot between each stroke. The dip motion takes about 10 seconds to complete, as the robot retracts the brush from the easel, moves down to dip and returns. Performing this operation between each of dozens of strokes therefore requires continuous robot motion for hours. From observing the motion we learned that it was unlikely that these motions were produced automatically. Although apparently repetitive, there were slight variations in the motion. We observed that the user occasionally changes the brush and that while doing so he or she pauses after grasping the brush to see, via camera, that the gripper has actually obtained the brush. If the robot failes to grasp the brush the user tries again, and this is a sophisticated maneuver that shows the user is observing the task and reacting to perceived conditions.

The following text was placed in the image gallery:

"To my disappointment, this no longer appears to be a sophisticated prankster. I stayed late and watched the AOL user closely while producing this highly over stroked image. The strokes are less regular than I originally thought, and the user clearly is online some of the time...."

At this time the robot began to have mechanical problems related to the rough use it was receiving. The end effector broke, requiring a week or so to repair, and the period offline apparently caused the AOL user to stay away for a while. The user returned next on January 20th, 2004, as shown in Figure 10. Again we see dozens of vertical and horizontal strokes resulting ultimately in the teardown of the canvas as shown in Figure 11. At this point the user seems to switch his or her empahsis from placing paint on the canvas to tearing the paper down and writing on the easel.

The AOL user becomes very good at this task over the next several days, and adds taunting messages to the easel after tearing down the paint. At one point during the painting I placed a placard on the easel asking "Just what are you trying to accomplish?" The AOL User did not respond to this question but did use the brushes to tear down the placard. The following text was posted in the image gallery:

"I can stop this any time I want, but its rather fun to watch. One interesting thing is that he or she has become the most advanced PumaPaint user in the history of this project. He or She shows remarkable aptitude in determining if the brush is acquired, righting it if it gets crooked (not often possible) and also knows that vertical strokes work better than horizontal. At this point he or she is a master at tearing down the paper. "

Although continuing this interaction would have been interesting, we were unable to do so because of mechanical problems in the robot. Likely caused directly or indirectly by the heavy usage, Joint 2 of the robot began to overheat and lost sufficient torque to lift the arm. Repair of the robot took over three months, and the AOL User has jus recently returned. One interesting aspect of this episode is that the AOL user never direclty responded to my requests for more information as to how he or she was accomplishing these repetitive motion commands. On the main page has always been an offer to mail canvases to users, and the AOL user did contact me, asking for the canvases that he or she had torn down. The email message was not signed and was sent from an AOL account. But a mailing address was provided so true to our word we sent a number of repetitively stroked and torn down canveses to a maling address in Hackettstown, New Jersey. During the repair period we did receive one more unsigned message from the AOL user rebuking us with a single-line message: "well you would think an engineering school could fix a robot in less time!"

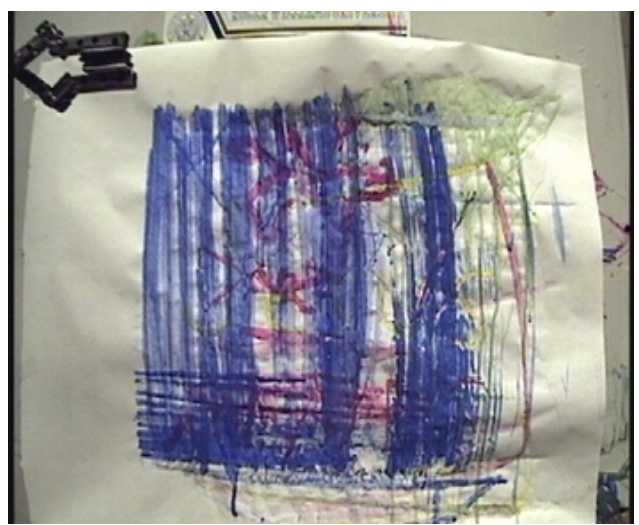

Figure 10. Vertical strokes successfully tearing down paper.

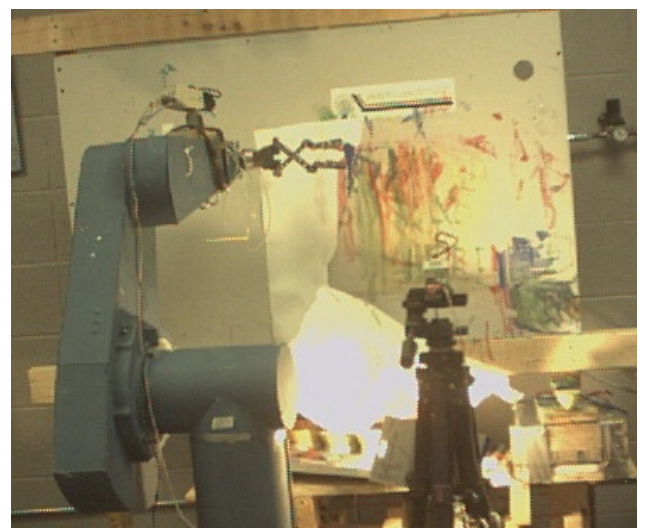

Figure 11. Successful teardown of the paper.

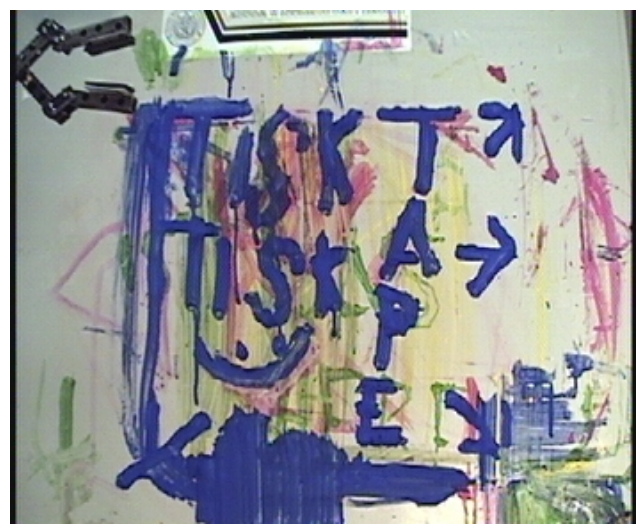

Figure 12. Successful teardown followed by taunting. 


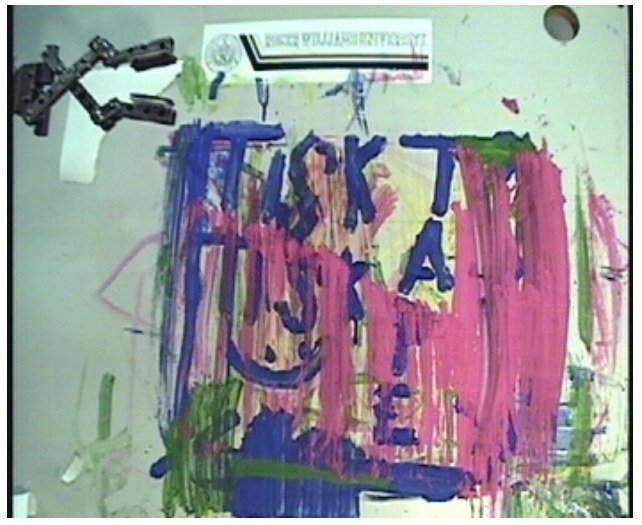

Figure 13. Illuminating vertical stroke pattern.

Figure 13 shows an interesting stroke pattern that provides a clue as to how the user is performing this task. We see a progression of vertical strokes from left to right with linearly decreasing length, and it is hard to imagine the user is sitting in front of their computer for half an hour meticulously placing these strokes on the virtual canvas. We we now believe the AOL user discovered that he or she could queue up hours of commands in relatively short time. We believe the AOL user was doing this by rapidly moving the mouse vertically over the virtual canvas, exceeding the ability of the java applet to keep up with mouse events. If the mouse is moving fast enough there may be one mouse event each time the mouse is waved over the virtual canvas, and this would generate single vertical stroke command rapidly accumulating in the command queue. The AOL user then sits back and watches the robot perform ten to thirty minutes of motion until the queue is empty. There is a limit on the output queue of 200 commands, so the AOL user cannot enqueue hours and hours of motion. By observation we could see the AOL user returning when the queue was empty to enqueue hundreds of more commands.

We had never seen a previous user manipulate the command queue in this manner. What has resulted is an educational experience in the nature of time-delayed teleroboticcs. The AOL user saw that the robot always trailed the operator actions and that the separation can be minutes or hours. Despite repeated requests, the AOL user has never identified him or herself or explained the purpose of their actions. We are left to speculate that the AOL user is probably a juvenile who lives in New Jersey and is just playing with the robot. We suspect the AOL user is a juvenile because he or she has spent hundreds of hours connected to the site, and what adult user has that kind of time on their hands?

\section{THE MOVE TO THE THIRD DIMENSION}

We are currently developing the next phase of the PumaPaint experience, allowing users to remotely model clay using a pair of roughly anthropomorphic hands. Each of the two "hands" will consist of three "fingers" each with one degree of freedom: a major index finger, a minor index finger, and a thumb (yet to be designed). These fingers will be driven by a motor system salvaged from a Rhino robot. The entire hand assembly will be attached to a linear positioning stage to supply forward and backward movement. Figure 14 shows the complete parametric Inventor ${ }^{\circledR}$ model, with the four designed fingers on a bracket in opposition.

\section{A. Major Index Finger}

The major index finger will be positioned at the top end of the "palm" area. It is a one-degree of freedom linkage system. There are six bar sections connected by fourteen joints. Each bar has been modeled using 5/8" square steel tubing. This tubing has a filleted hollow square cross section with an inner wall length of $1 / 2$ ". Two of the sections are made of welded bar sections. Each joint is a flanged bearing inserted into a drilled hole at both sides of the bar stock. A 1/8" steel rod is then inserted through all bearings making up a passive revolute joint.

We intended the overall motion to closely duplicate the motion of a human index finger based on physiological measurements presented in [7]. In Figure 15, the opaque image represents the initial position of the assembly. The images get less opaque towards the end of the motion direction ending with another opaque image.

The motion of the major index finger was then used to design the minor index finger. We wanted the minor index finger to mimic the motion of the major index finger in the middle of its path. Also the minor index finger had to break out of synchronization with the major index finger when it was at the beginning and end of its motion path. This would best reflect the motion of the human hand

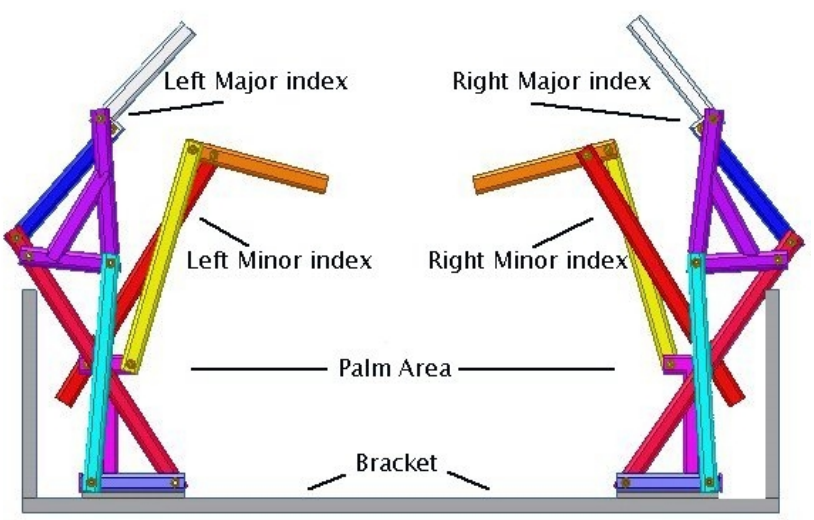

Figure 14. Robotic hand substructure

\section{B. Sensors}

A sensory system is essential to the task that this manipulator will perform. However, we need to balance the amount of sensing we provide the user with the amount of space we have and our limited fabrication ability. The sensory system has the primary purpose of telling the user if the robot is in contact with the clay, and if so the pressure of contact. We decided to employ a relatively simple sensor system that has three states: "off" state (not touching clay), low pressure touching, and high pressure touching.

Our goal is to give the user useful touch sensor data that enhances usability of the hands. It could be argued that a higher number of sensory states could give a user an even 
greater control. However, this adds complexity to the design, and it is uncertain that this complexity is warranted. We are ultimately limited in our ability to communicate pressure information by the capacity of the communication channel and the ability to display haptic information in the presence of communication time delay. Even with haptic display, (e.g. a phantom device at the user station) the time delay in communication limits the haptic communication to symbolic presentation. It makes little sense to design more pressure sensing instrumentation into the hands than can be effectively communicated to the remote user. We recognize that this is an open research issue and we may find more pressure states are necessary for useful haptic information.

We will implement contact sensing with appropriately placed optical sensors. Each part in the assembly will be encased in a semi-circular section of polymer piping cut longitudinally, as shown in Figure 16. These semicircular cylinders will be secured to an aluminum channel affixed around the steel tubing section of each part. A compliant foam pad will be inserted under each piece of channel. This will allow each polymer section to move compliantly. Two optical sensors will be installed within the steel tubing. When pressure is applied the first optical sensor will be triggered, when more pressure is applied the second one will be triggered.

\section{SUMMARY AND CONCLUSIONS}

The PumaPaint site has provided an artistic experience aided by a remote robotic device to roughly 15,000 users over the last six years. Although we make no claims that connecting a PUMA robot to the Internet is particularly challenging, keeping it running this long has taken, if nothing else, perseverance. We would claim that we have made a worthy contribution to networked robotics simply by keeping this site operational.

Figure 17 shows a canvas created the week this famous piece was stolen, and this makes it difficult to contemplate shutting down the PumaPaint site. We anticipate these two experiences will coexist for some time. We expect to offer both alternatives, and hopefully measure the relative appeal of the two experiences. We are hoping to make more progress assembling the mechanical components this fall and optimistically anticipate some form of the clay modeling experience to online by late spring, 2005.

\section{REFERENCES}

[1] http://pumapaint.rwu.edu

[2] M. R. Stein, C Ratchford, K. Sutherland, D. Robaczewski, "Internet Robotics: An Educational Experiment", Telemanipulator and Telepresence Technologies II, SPIE Volume 2590, 1995

[3] M. R. Stein, K. Sutherland, "Sharing Resources over the Internet for Robotics Education", Telemanipulator and Telepresence Technologies IV, SPIE Volume 3206, 1997

[4] P. DePasquale, J. Lewis, M. R. Stein, "A Java Interface for Asserting interactive Telerobotic Control", Telemanipulator and Telepresence Technologies IV, SPIE Volume 3206, 1997

[5] Matthew R. Stein, "The PumaPaint Project", Journal of Autonomous Robots, Fall 2003

[6] http://yugo.mme.wilkes .edu/ villanov
[7] Gongliang Guo, Tsu-Tian Lee, William Gruver and Jichuan Zhang, "Design of a Planar Multijointed Prosthetic Finger Mechanism", $21^{s t}$ ASME Mechanisms Conference DE-Vol 26, pp 165-170, 1990

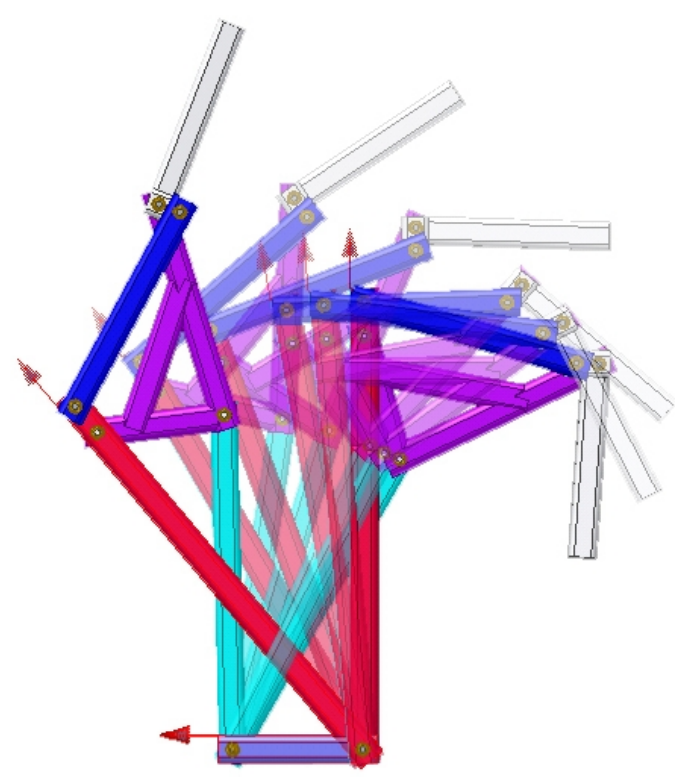

Figure 15. Range of motion of major index finger
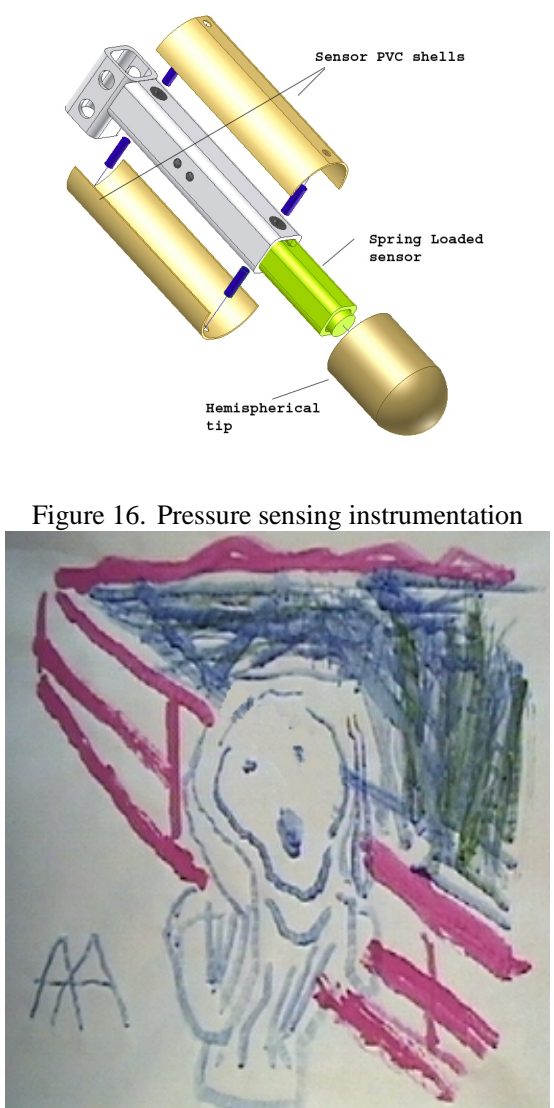

Figure 17. Munch painting found. 